F. S. Cater, Department of Mathematics, Portland State University, Portland, Oregon 97207, USA

\title{
ON THE DERIVATIVES OF FUNCTIONS OF BOUNDED VARIATION
}

\begin{abstract}
Using a standard complete metric $w$ on the set $F$ of continuous functions of bounded variation on the interval $[0,1]$, we find that a typical function in $F$ has an infinite derivative at continuum many points in every subinterval of $[0,1]$. Moreover, for a typical function in $F$, there are continuum many points in every subinterval of $[0,1]$ where it has no derivative, finite nor infinite. The restriction of the derivative of a typical function in $F$ to the set of points of differentiability has infinite oscillation at each point of this set.
\end{abstract}

Let $C[0,1]$ denote the family of continuous real valued functions on the interval $[0,1]$ and let $F$ denote the set of functions of bounded variation in $C[0,1]$.

It is known (see for example $[\mathrm{B}]$ or $[\mathrm{C}]$ ) that with respect to the uniform metric on $C[0,1]$, a typical function in $C[0,1]$ has a unilateral infinite derivative at continuum many points in each subinterval of $[0,1]$, even though it has no finite unilateral derivative at any point. We wondered if some sort of analogue can be constructed for $F$. Problems of finding such an analogue are two-fold: the uniform metric is not complete on $F$, and functions in $F$ are differentiable almost everywhere. So we define

$$
w(f, g)=|f(0)-g(0)|+\text { total variation of } f-g \text { on }[0,1] .
$$

The proof that $w$ is a complete metric on $F$ is well-known (see [R, p. 147], for example).

With respect to the metric $w$, we will show that a typical function in $F$ has infinite derivatives at continuum many points in each subinterval of $[0,1]$. For any residual set $S$, we will find that a typical $f \in F$ satisfies $f^{\prime}(x) \in S$ almost

Key Words: bounded variation, absolutely continuous, singular, derivative, complete metric, category.

Mathematical Reviews subject classification: 26A21, 26A24, 26A27, 26A30, 26A45, 26A46, 26A 48.

Received by the editors December 28, 2000 
everywhere. For any subset $E$ of $(0,1)$, with exterior measure 1 , we will show that the restriction to $E$ of the Dini derivatives of any typical function in $F$ are discontinuous at each point of $E$. All derivatives here are two-sided.

Theorem I. For a typical $f \in F$, the set

$$
\left\{x \in I:\left|f^{\prime}(x)\right|=\infty\right\}
$$

has the power of the continuum for each subinterval I of $[0,1]$.

Proof. Let $[c, d]$ be a subinterval of $[0,1]$. Let $k \in F$ and let $\epsilon$ be a positive number. Choose a subinterval $[a, b]$ of $[c, d]$ such that

$$
V(k,[a, b])<\frac{\epsilon}{8} .
$$

(Here $V$ denotes total variation.) Let $f$ be a singular nondecreasing function in $F$, that vanishes on $[0, a]$, is constant on $[b, 1]$ and such that

$$
f(b)-f(a)=\frac{\epsilon}{2} .
$$

(Lebesgue's singular function can be used to construct $f$; see [HS, (8.28)].) Then $w(k+f, k)=\epsilon / 2$.

Now any function in the open ball with center $k+f$ and radius $\epsilon / 8$ can be expressed $k+f+g$ where $g \in F$ and $w(g, 0)<\epsilon / 8$. Then

$$
V(k+g,[a, b]) \leq V(k,[a, b])+V(g,[a, b])<\frac{\epsilon}{8}+w(g, 0)<\frac{\epsilon}{8}+\frac{\epsilon}{8}=\frac{\epsilon}{4},
$$

and

$$
V(k+g,[a, b])<\frac{\epsilon}{2}=f(b)-f(a) .
$$

It follows from this and the fact that $f$ is singular on $[a, b]$, that $k+f+g$ is not absolutely continuous on $[a, b]$ nor on $[c, d]$. Thus the set of functions in $F$ that are not absolutely continuous on $[c, d]$ form a residual subset of $F$.

Finally, let $[c, d]$ run over all the subintervals of $[0,1]$ with rational endpoints and find that the set of functions in $F$ that are absolutely continuous on no subinterval of $[0,1]$ form a residual subset of $F$. But such functions must have infinite derivatives at continuum many points in each subinterval of $[0,1]$.

Theorem II. For every residual set of real numbers $S, f^{\prime}(x) \in S$ almost everywhere for typical $f \in F$ (in particular, for such sets $S$ of measure 0 ). 
Proof. Let $p$ be a positive number and let $X$ be a closed nowhere dense subset of $\mathbb{R}$. It suffices to prove that the set of all $g \in F$ for which

$$
m\left\{x \in(0,1): g^{\prime}(x) \in X\right\} \geq p
$$

is a nowhere dense subset of $F$.

So let $T$ denote the set of all $g \in F$ for which $m\left\{x \in(0,1): g^{\prime}(x) \in\right.$ $X\} \geq p$. Let $k \in F \backslash T$. Then

$$
m\left\{x \in(0,1): k^{\prime}(x) \in X\right\}<p .
$$

There are positive numbers $r$ and $q$ such that

$$
m\left\{x \in(0,1): \text { the distance from } k^{\prime}(x) \text { to } X \text { is less than } q\right\}=r<p .
$$

Choose any $h \in T$. Then

$$
m\left\{x \in(0,1):\left|k^{\prime}(x)-h^{\prime}(x)\right| \geq q\right\} \geq p-r .
$$

We apply the Vitali Covering Theorem to this set to find mutually disjoint intervals $\left[x_{i}, u_{i}\right]$ such that

$$
\sum_{i}\left(u_{i}-x_{i}\right) \geq p-r
$$

and for each index $i$,

$$
\left|(k-h)\left(u_{i}\right)-(k-h)\left(x_{i}\right)\right| \geq \frac{q\left(u_{i}-x_{i}\right)}{2} .
$$

Consequently,

$$
\sum_{i}\left|(k-h)\left(u_{i}\right)-(k-h)\left(x_{i}\right)\right| \geq \frac{q(p-r)}{2} .
$$

It follows that

$$
w(h, k) \geq \frac{q(p-r)}{2},
$$

and $T$ is a closed subset of $F$. It remains to prove that $F \backslash T$ is dense in $F$.

Let $\epsilon$ be a positive number. Let $\left(y_{j}\right)_{j=-\infty}^{\infty} \subset \mathbb{R} \backslash X$ be a sequence such that

$$
\lim _{j \rightarrow-\infty} y_{j}=-\infty, \quad \lim _{j \rightarrow \infty} y_{j}=\infty \quad \text { and } \quad 0 \leq y_{j}-y_{j-1}<\epsilon \text { for each } j .
$$


Let $h_{0} \in F$. For $x \in[0,1]$, define

$$
f_{1}(x)=r_{j}-h_{0}^{\prime}(x) \text { where } j \text { is such that } r_{j}>h_{0}^{\prime}(x) \geq r_{j-1} .
$$

Then $0 \leq f_{1}(x)<\epsilon$. Let $f_{2}$ be the indefinite integral of $f_{1}$ :

$$
f_{2}(x)=\int_{0}^{x} f_{1}(t) d t
$$

Then $0 \leq f_{2}^{\prime}(x) \leq \epsilon$ almost everywhere and

$$
w\left(f_{2}, 0\right)=V\left(f_{2},[0,1]\right)=\int_{0}^{1} f_{2}^{\prime}(t) d t \leq \epsilon .
$$

Also $f_{2}^{\prime}(x)+h_{0}^{\prime}(x)$ is in the set $\left\{r_{j}\right\} \subset \mathbb{R} \backslash X$ almost everywhere, so $f_{2}+h_{0} \notin T$. Finally

$$
w\left(f_{2}+h_{0}, h_{0}\right)=w\left(f_{2}, 0\right) \leq \epsilon .
$$

Thus $F \backslash T$ is a dense open subset of $F$.

Theorem III. Let $E$ be any subset of $[0,1]$ with exterior measure 1 . Then the restriction to $E$ of the Dini derivates of a typical function in $F$ are discontinuous on E. Moreover, their oscillations at each point of $E$ are infinite.

Proof. Let $I$ be an open subinterval of $[0,1]$ and $J$ be an open subinterval of $\mathbb{R}$. Then it suffices to prove that the set of functions $g \in F$ for which

$$
m\left\{x \in I: g^{\prime}(x) \in J\right\}>0
$$

is an open dense subset of $F$. Let $T$ denote the set of all $g \in F$ for which $m\left\{x \in I: g^{\prime}(x) \in J\right\}=0$. Take $h \notin T$. Then $m\left\{x \in I: h^{\prime}(x) \in J\right\}>0$. Let $s$ and $r$ be positive numbers such that

$$
m\left\{x \in I \text { : the distance from } h^{\prime}(x) \text { to } \mathbb{R} \backslash J \text { is at least } s\right\}=r>0 .
$$

Let $g \in T$. So

$$
m\left\{x \in I:\left|h^{\prime}(x)-g^{\prime}(x)\right| \geq s\right\} \geq r .
$$

We use the Vitali Covering Theorem on this set to find pairwise disjoint intervals $\left[a_{i}, b_{i}\right]$ such that

$$
\left|(h-g)\left(b_{i}\right)-(h-g)\left(a_{i}\right)\right| \geq \frac{s\left(b_{i}-a_{i}\right)}{2} \text { for each } i
$$


and $\sum_{i}\left(b_{i}-a_{i}\right) \geq r$. Hence

$$
w(h, g) \geq V(h-g,[0,1]) \geq s \sum_{i} \frac{b_{i}-a_{i}}{2} \geq \frac{r s}{2},
$$

and $T$ is a closed subset of $F$.

Now let $g_{0} \in T$, and let $p$ be a positive number. Let $[a, b]$ be a subinterval of $I$ for which $V\left(g_{0},[a, b]\right)<p / 4$. It is easy to construct a function $g_{1} \in F$ that coincides wit $g_{0}$ on $[0, a]$ and on $[b, 1]$, for which

$$
V\left(g_{1},[a, b]\right)<\frac{p}{2} \quad \text { and } m\left\{x \in[a, b]: g_{1}^{\prime}(x) \in J\right\}>0 .
$$

Hence $g_{1} \notin T$ and

$$
w\left(g_{0}, g_{1}\right)=V\left(g_{1}-g_{0},[a, b]\right) \leq V\left(g_{1},[a, b]\right)+V\left(g_{0},[a, b]\right)<\frac{p}{2}+\frac{p}{4}<p .
$$

So $T$ is a nowhere dense closed set.

Note that the set $F_{1}$ of nondecreasing functions in $F$ is a closed subset of $F$. So $F_{1}$ is a complete metric space under $w$ in its own right. The Theorems I, II and III are also true with $F_{1}$ in place of $F$ by essentially the same arguments.

Let $g \in F_{1}$ and assume that $D^{+} g<\infty$ on a second category subset of $[0,1]$. It follows that there is a second category set $E$ such that the set

$$
\left\{\frac{g(x+p)-g(x)}{p}: p>0, x \in E\right\}
$$

is bounded. Let $I$ be a subinterval of $[0,1]$ in which $E$ is dense. By continuity, the difference quotient of $g$ is bounded on $I$. On the other hand, it is easy to prove that the set of all functions in $F_{1}$ with bounded difference quotient on $I$ is a first category subset of $F_{1}$. It follows that the set of all $g \in F_{1}$ such that $D^{+} g(x)=\infty$ on a residual subset of $[0,1]$ is a residual subset of $F_{1}$. Likewise it is easy to prove that the set of all functions in $F_{1}$ with difference quotient bounded away from 0 on $I$ is a first category subset of $F_{1}$. By an analogous argument it follows that the set of all $g \in F_{1}$ such that $D_{+} g(x)=0$ on a residual subset of $[0,1]$ is a residual subset of $F_{1}$. The corresponding statements can be proved for $D^{-} g$ and $D_{-} g$. We conclude with:

Proposition 1. For a typical $f \in F_{1}$, the set

$$
\left\{x \in(0,1): D^{+} f(x)=D^{-} f(x)=\infty \text { and } D_{+} f(x)=D_{-} f(x)=0\right\}
$$

is a residual subset of $[0,1]$. Thus typical $f \in F_{1}$ have unilateral derivatives, finite or infinite, on at most a first category subset of $[0,1]$. 
For $f \in F_{1}$ we define the four sets:

- $A_{f}=\left\{x \in(0,1): D_{-} f(x)=D_{+} f(x)=0\right\}$,

- $B_{f}=\left\{x \in(0,1): D^{-} f(x)=D^{+} f(x)=\infty\right\}$,

- $C_{f}=\left\{x \in(0,1): D_{-} f(x)=0\right.$ and $\left.D^{+} f(x)=\infty\right\}$,

- $D_{f}=\left\{x \in(0,1): D^{-} f(x)=\infty\right.$ and $\left.D_{+} f(x)=0\right\}$.

(The idea is that in each set there is one restriction on the left and one on the right.) For typical $f \in F_{1}$, we know that $A_{f} \cup B_{f} \cup C_{f} \cup D_{f}$ is a residual subset of $[0,1]$.

Is there a strictly increasing singular function $f$ for which $A_{f} \cup B_{f} \cup C_{f} \cup D_{f}=$ $(0,1)$ ? The answer is yes; we showed how one can be constructed in [C1].

Is there a strictly increasing singular function in $F_{1}$ for which $(0,1)$ equals the union of any three of these sets? The answer, we shall see, is no.

Proposition 2. Let $f$ be a strictly increasing singular function in $F_{1}$. Then each of the sets

$$
A_{f} \cup B_{f} \cup C_{f}, \quad A_{f} \cup B_{f} \cup D_{f}, \quad A_{f} \cup C_{f} \cup D_{f}, \quad B_{f} \cup C_{f} \cup D_{f},
$$

has a dense complement in $[0,1]$.

Proof. Let $I$ be a subinterval of $[0,1]$. Because $f$ is a singular function, we deduce that there exist points $a, b \in I$ such that

$$
a<b, \quad f^{\prime}(a)=\infty \quad \text { and } \quad f^{\prime}(b)=0 .
$$

Let $G$ denote the graph in $\mathbb{R}^{2}$

$$
\{(x, f(x)): a \leq x \leq b\} .
$$

Then $G$ is a compact subset of $\mathbb{R}^{2}$. Let $r$ be the maximum value for which the line (in $\mathbb{R}^{2}$ ) $y=x+r$ meets $G$. Say they meet at the point $(u, f(u))$. By comparing the slope of the line with the slope of the graph, we conclude that $u \neq a$ and $u \neq b$. So $a<u<b$ and $u \in I$. By the same reasoning we find that $D^{+} f(u) \leq 1$ and $D_{-} f(u) \geq 1$. It follows that $u \notin A_{f} \cup B_{f} \cup C_{f}$. By the analogous argument (with $b<a$ and $r$ minimal) we find a point in $I$ that is not in $A_{f} \cup B_{f} \cup D_{f}$. Of course any point where $f^{\prime}=\infty$ is not in $A_{f} \cup C_{f} \cup D_{f}$ and any point where $f^{\prime}=0$ is not in $B_{f} \cup C_{f} \cup D_{f}$. The conclusion follows.

Let us recapitulate. For typical $f \in F_{1}$ and any subinterval $I$ of $[0,1]$ we have: 
1) $f$ has derivative $\infty$ at continuum many points in $I$,

2) $f$ has a finite derivative at continuum many points in $I$,

3) there are continuum many points in $I$ at which $f$ has no derivative, finite or infinite,

4) the restriction of $f^{\prime}$ to the set of all points of differentiability of $f$, has infinite oscillation at each point.

We conclude by giving an indirect but elementary proof of the well-known result that the set

$$
\left\{x \in(a, b):\left|f^{\prime}(x)\right|=\infty\right\}
$$

has measure zero. The arguments will not require the Vitali Covering Theorem nor the differential properties of monotonic functions. We require only the following well-known facts, that we state without proof.

Lemma A. If $S_{1}, S_{2}, S_{3}, \ldots$ are finitely many subsets of $[a, b]$, then

$$
\sum_{i} m\left(S_{i}\right) \geq m\left(\cup_{i} S_{i}\right)
$$

where $m$ denotes Lebesgue outer measure.

Lemma B. If $S_{1}, S_{2}, S_{3}, \ldots$ is a sequence of subsets of $[a, b]$, then there is an index $k$ such that

$$
m\left(\cup_{i=1}^{k} S_{i}\right) \geq \frac{1}{2} \cdot m\left(\cup_{i=1}^{\infty} S_{i}\right) .
$$

PROOF OF THE RESULT. It suffices to prove the result for bounded functions. Then it will hold for arbitrary functions by truncating such a function at $N$ and $-N$. So let $g$ be a bounded function on $[a, b]$ and let $E \subset[a, b]$ be a set such that $g^{\prime}(x)=\infty$ at each $x \in E$. The plan is to assume that $m(E)>0$ and eventually find a contradiction. Fix an integer $N$ so large that on $[a, b]$

$$
N>2 \cdot|g|
$$

So

$$
E=\bigcup_{j=1}^{\infty}\left\{x \in E: \frac{f(x)-f(u)}{x-u}>\frac{8 N}{m(E)} \text { for } 0<|x-u|<\frac{1}{j}\right\} .
$$

By Lemma $\mathrm{B}$, there is an index $k$ for which $m\left(E_{1}\right)>m\left(E_{2}\right) / 2$ where

$$
E_{1}=\left\{x \in E: \frac{g(x)-g(u)}{x-u}>\frac{8 N}{m(E)} \text { for } 0<|x-u|<\frac{1}{k}\right\} .
$$


Choose points $u_{0}, u_{1}, u_{2}, \ldots, u_{p}$ such that

$$
a=u_{0}<u_{1}<u_{2}<\ldots<u_{p}=b \text { and } u_{i}=u_{i-1}<\frac{1}{k} \text { for } i=1,2, \ldots, p .
$$

For each index $i$ for which the interval $\left[u_{i-1}, u_{i}\right]$ meets $E_{1}$, choose a point $x_{i} \in$ $\left[u_{i-1}, u_{i}\right] \cap E_{1}$ such that $2\left(u_{i}-x_{i}\right)$ exceeds the diameter of the set $\left[u_{i-1}, u_{i}\right] \cap E_{1}$. Then

$$
u_{i}-x_{i}>\frac{1}{2} \cdot m\left(\left[u_{i-1}, u_{i}\right] \cap E_{1}\right) .
$$

We sum over the indices $i$ for which $\left[u_{i-1}, u_{i}\right] \cap E_{1}$ is nonvoid and obtain (by Lemma A)

$$
\sum_{i}\left(u_{i}-x_{i}\right)>\sum_{i} \frac{1}{2} \cdot m\left(\left[u_{i-1}, u_{i}\right] \cap E_{1}\right)>\frac{1}{2} \cdot m\left(E_{1}\right) .
$$

But $m\left(E_{1}\right)>\frac{1}{2} \cdot m(E)$, so

$$
\sum_{i}\left(u_{i}-x_{i}\right)>\frac{1}{4} \cdot m(E)
$$

By the definition of $E_{1}$,

$$
\sum_{i}\left(g\left(u_{i}\right)-g\left(x_{i}\right)\right)>\sum_{i} \frac{8 N}{m(E)} \cdot\left(u_{i}-c_{i}\right)=\frac{8 N}{m(E)} \cdot \sum_{i}\left(u_{i}-x_{i}\right)
$$

and by (2),

$$
\sum_{i}\left(g\left(u_{i}\right)-g\left(x_{i}\right)\right)>\frac{8 N}{m(E)} \cdot \frac{m(E)}{4}=2 N .
$$

Note also that no two of the intervals $\left[x_{i}, u_{i}\right]$ overlap.

From Lemma A we deduce that one of the sets $\left[u_{i-1}, u_{i}\right] \cap E_{1}$ does not have measure zero; call this set $E_{2}$. Thus there is a subinterval $[c, d]$ of $[a, b]$, containing this subset $E_{2}$ of $E_{1}$, such that

$$
d-c<\frac{1}{k}, \quad m\left(E_{2}\right)>0, \quad \text { and } \quad g^{\prime}(x)=\infty \text { for each } x \in E_{2}
$$

We repeat the preceding arguments with $[c, d]$ in place of $[a, b]$ and $E_{2}$ in place of $E$, to find mutually nonoverlapping subintervals

$$
\left[y_{1}, v_{1}\right],\left[y_{2}, v_{2}\right],\left[y_{3}, v_{3}\right], \ldots,\left[y_{t}, v_{t}\right]
$$


of $[c, d]$, such that $y_{j} \in E_{2}$ for all $j$ and

$$
\sum_{j=1}^{t}\left(g\left(v_{j}\right)-g\left(y_{j}\right)\right)=2 N .
$$

We index these intervals so that $y_{1} \leq y_{2} \leq y_{3} \leq \ldots \leq y_{t}$. Because the intervals do not overlap, we have in fact

$$
y_{1}<v_{1} \leq y_{2}<v_{2} \leq y_{3}<v_{3} \leq \ldots \leq y_{t}<v_{t} .
$$

But each $y_{j} \in E_{1}$ also, and we deduce from the definition of $E_{1}$ that $v_{j}-y_{j}$ and $g\left(v_{j}\right)-g\left(y_{j}\right)$ are both positive, and $y_{j}-v_{j-1}$ and $g\left(y_{j}\right)-g\left(v_{j-1}\right)$ are both nonnegative. It follows from (5) that

$$
g\left(y_{1}\right)<g\left(v_{1}\right) \leq g\left(y_{2}\right)<g\left(v_{2}\right) \leq g\left(y_{3}\right)<g\left(v_{3}\right) \leq \ldots \leq g\left(y_{t}\right)<g\left(v_{t}\right) .
$$

From (4) and (6) we obtain

$$
g\left(v_{t}\right)-g\left(y_{1}\right) \geq \sum_{j=1}^{t}\left(g\left(v_{j}\right)-g\left(y_{j}\right)\right)>2 N .
$$

By (1) we have $g\left(v_{t}\right)-g\left(y_{1}\right)<N$. Combining this with (7), we have

$$
N>2 N \text {. }
$$

Finally, $0>N$ contrary to (1). This contradiction completes the proof.

\section{References}

[B] A. Bruckner, Some new simple proofs of old difficult theorems, Real Anal. Exchange, 9 (1984), 74-75.

[C] F. S. Cater, An elementary proof of a theorem on unilateral derivatives, Can. Math. Bull., 29 (3), (1986), 341-343.

[C1] F. S. Cater, On the Dini derivatives of a particular function, Real Anal. Exchange, 25 (1), (1999/2000), 943-946.

[HS] E. Hewitt and K. Stromberg, Real and Abstract Analysis, SpringerVerlag, New York, 1965.

[R] W. O. Ray, Real Analysis, Prentice Hall, Englewood Cliffs, 1988.

[S] S. Saks, Theory of the integral, 2nd rev. ed., Dover, New York, 1964. 
F. S. CATER 